\title{
Detection of Rotavirus Strains in Freshwater Clams in Japan
}

\author{
Sheikh Ariful Hoque ${ }^{1,2} \cdot$ Azumi Wakana $^{3} \cdot$ Hideaki Shimizu $^{3} \cdot$ Sayaka Takanashi $^{4} \cdot$ Shoko Okitsu $^{1}$. \\ Kazi Selim Anwar ${ }^{5,6}$. Satoshi Hayakawa ${ }^{1} \cdot$ Niwat Maneekarn $^{7} \cdot$ Nobuhiko Okabe $^{3} \cdot$ Hiroshi Ushijima ${ }^{1}$ (])
}

Received: 7 June 2021 / Accepted: 1 December 2021 / Published online: 3 January 2022

(c) The Author(s), under exclusive licence to Springer Science+Business Media, LLC, part of Springer Nature 2022

\begin{abstract}
Bivalve molluscan shellfish like clams and oysters, etc., are capable to bioaccumulate surrounding contaminants from waters into their digestive systems and posing serious threats of food poisoning. Detection of rotaviruses (RVs) in shellfish is of particular importance because RVs are prone to genome reassortment resulting in the emergence of new RV variants that may compromise vaccine safety. Herein, we have detected the wild-type RVs and Rotarix/RotaTeq vaccine strains in freshwater clams collected on the riverside, Kawasaki city, from July 2019 to January 2020 and correlated the detected genotypes with that of gastroenteritis cases of nearby clinics to understand the transmission of RVs in the environment. The wild-type RVs were detected in 62 (64.6\%) out of 96 freshwater clams in every study month: July, September, November, and January that are considered as off-season for RV infections. The most frequent genotypes were G2 (42.9\%), G8 (28.6\%), G3 (14.3\%), G1 (7.1\%), and G10 (7.1\%), which remained comparable with genotypic distribution found in the clinical samples over the last few years indicating that these RVs may accumulate in clams since a long time. However, G10 genotype was detected in clam but not in clinical samples suggesting the presence of asymptomatic infection or RVs could be carried out from a long distance. Importantly, vaccine strains, RotaTeq (1\%) but not Rotarix (0\%), were also detected in a clam. Attention must be paid to monitoring the potential transmission of wild-type and vaccine RV strains in the environment to prevent the emergence of new variants generated from genome reassortment with vaccine strains.
\end{abstract}

Keywords Rotavirus $\cdot$ Vaccine strains $\cdot$ Clams $\cdot$ Environmental samples $\cdot$ Clinical samples

Hiroshi Ushijima

ushijima-hiroshi@jcom.home.ne.jp

1 Division of Microbiology, Department of Pathology and Microbiology, Nihon University School of Medicine, 30-1 Oyaguchi Kamicho, Itabashi-ku, Tokyo 173-8610, Japan

2 Cell and Tissue Culture Laboratory, Centre for Advanced Research in Sciences (CARS), University of Dhaka, Dhaka, Bangladesh

3 Kawasaki City Institute for Public Health, Kawasaki City, Kanagawa, Japan

4 Infectious Disease Surveillance Center, National Institute of Infectious Diseases, Shinjuku-Ku, Tokyo, Japan

5 Ad-Din Women Medical College Hospital (AWMCH), Dhaka, Bangladesh

6 Department of Public Health, Daffodil International University (DIU), Dhaka, Bangladesh

7 Department of Microbiology, Faculty of Medicine, Chiang Mai University, Chiang Mai, Thailand

\section{Introduction}

Rotavirus (RV) still remains the most common cause of severe diarrhea among children under 5 years of age that could be fatal due to serious dehydration caused by extensive diarrhea and vomiting (Araki et al., 2016; WHO, 2019). To prevent severe RV illness, two live attenuated oral RV-vaccines, Rotarix (GlaxoSmithKline, Rixensart, Belgium) and RotaTeq (Merck \& Co., Whitehouse Station, NJ, USA) were recommended by World Health Organization (WHO) to be introduced in every country since 2009 (WHO, 2019). Yet, $\mathrm{RV}$ s were found responsible, mostly in developing countries, for an estimated 258 million diarrheal cases and 128,515 deaths annually worldwide among children $<5$ years while 228,047 deaths among all ages (GBD, 2016 Diarrhoeal Disease Collaborators, 2018). However, the death toll remained as high as 453,000 among children younger than 5 years in 2008 when RV-vaccines were not implemented widely (Tate et al., 2012; WHO, 2019). In fact, RV-vaccines played a dramatic role in reducing disease severity, hospitalizations, 
and mortality in RV infection through providing a broad heterotypic immunity because of RV's cross-reactivity (Burnett et al., 2017; Hoque et al., 2018a, 2018b; Kawata et al., 2021). However, RV infection yet remains unpreventable not only because of RV's genotype diversity and reassortment capability but also for its highly contagious nature (Hoque et al., 2018a, 2018b, 2019a, 2019b). Not only children but also adults could be infected and can spread the virus though the illness may remain less severe or asymptomatic in adults (Anses, 2012; Snelling et al., 2011). A large number of RVs are shed through the stool of infected persons and spread out easily via contaminated food, unwashed hands, and contaminated inanimate objects like toys, towels, etc. (Stanford Children's Health, 2021; Victoria et al., 2014).

Bivalve molluscan shellfish like clams and oysters are commonly eaten raw or undercooked are capable to accumulate enteric viruses from their surrounding waters and bioconcentrate them within their digestive systems and, thus, have been reported for some large outbreaks of enteric viruses like norovirus and hepatitis A (Meghnath et al., 2019; Richards, 2016; Upfold et al., 2021). Detection of RVs in sewage water (Hoque et al., 2019a, 2019b; Thongprachum et al., 2018) and in shellfish (Ito et al., 2021; Keller et al., 2019; Kittigul et al., 2014; Quiroz-Santiago et al., 2014) has been shown scattered in some studies. However, the seasonal variation of $\mathrm{RV}$ detection in shellfish or its association with gastroenteritis epidemics is not yet clear. Particularly, the detection of RV-vaccine strains in shellfish has been barely reported.

The current national immunization program in Japan includes BCG, PCV (Pneumococcal Vaccine), DTP-IPV (Diphtheria, Tetanus and Pertussis and Injectable Polio), MR (Measles and Rubella), Japanese Encephalitis, chickenpox, Hib, HPV, hepatitis B, while rotavirus (Rotarix from November 2011 and RotaTeq from July 2012), seasonal influenza, mumps, hepatitis A, meningococcal vaccines were introduced as voluntary vaccination (JHI, 2021). Recently, rotavirus vaccines ( 2 doses of Rotarix or 3 doses of RotaTeq) have been included in the national vaccination program of Japan from October 1, 2020 (Kawata et al., 2021). The average $\mathrm{RV}$-vaccine coverage was $47.6 \%$ until the end of 2014 which has been increased gradually in the following years (Kawata et al., 2021). Rotarix is administered in 2 oral doses: 1st at 6-14 weeks and with 4 weeks' interval 2nd within 24 weeks, while RotaTeq is administered in 3 oral doses: 1st at 6-14 weeks and with 4 weeks' interval from each 2 nd and 3 rd doses by 32 weeks. These are live vaccines that can replicate in vaccinees and are shed in feces which is more common after the first dose from 1 to 15 days post-vaccination (Anderson, 2008). This shedding could be prolonged as long as 360 days after the last dose in immunocompromised patients (Patel et al., 2010). RV-vaccine strains have been shown highly stable in the aquatic environment
(Gautam et al., 2014). Therefore, shedding of the vaccine viruses may cause transmission of live vaccine viruses from vaccinated to unvaccinated children resulting in a potential threat of vaccine-associated diseases, especially, in immunocompromised individuals (Yen et al., 2011). Previously, RotaTeq vaccine-acquired RV infection was reported among children with severe combined immunodeficiency disorder (Werther et al., 2009). Again, vaccine viruses may reassort with other strains to produce reassortants that may remain associated with acute gastroenteritis (AGE) (Gautam et al., 2014). Such reassortants derived from the RotaTeq vaccine strain and wild-type human RV strains have been detected in AGE cases (Bucardo et al., 2012). Accumulation of RVvaccine viruses in shellfish thus possess potential health risk and needs to be investigated.

In this study, we intended to detect wild-type RVs and $\mathrm{RV}$-vaccine viruses in freshwater clams (Cyrenidae) collected in different seasons from the Tokyo Bay area near Kawasaki City Institute for Public Health. The genotypes of RVs in clams, in relation to genotypes in RV-associated gastroenteritis cases detected in Kawasaki City Institute for Public Health of two sentinel clinics, were also investigated.

\section{Methods}

The freshwater clams (Cyrenidae) of size about 3-4 cm long were collected from the Tokyo Bay area near Kawasaki City Institute for Public Health at the riverside of the mouth of the Tama River with 2 months' interval from July 2019 to January 2020. These clams were natural and rare that were collected with permission. Therefore, a limited number of clams were collected for investigation. In each study month, 24 clams were collected at a time from the same area. The clams were kept on ice and transferred to the laboratory where these were shucked and the digestive tissues were excised from clams. These were then stored into microtubes at $-80{ }^{\circ} \mathrm{C}$ until analysis.

For virus extraction from the clams, we followed the standard method as instructed in Food hygiene inspection guidelines: Standard methods of analysis in food safety regulation (JFHA, 2015). In brief, the mid-gut digestive tissue was excised from a single clam and taken in a tube, weighed, added 9 times weight of $\alpha$-amylase $(2.8 \mathrm{mg} / \mathrm{ml}$ in PBS), and crushed well with a bio-masher. Since we aimed to detect wild-type/vaccine RV strain from individual clams, we did not use the pooling method here. It was then incubated for $1 \mathrm{~h}$ at room temperature and centrifuged at $10,000 \mathrm{rpm}$ at $4{ }^{\circ} \mathrm{C}$ for $20 \mathrm{~min}$. The supernatant was collected carefully and mixed with an equal volume of polyethylene glycol (PEG) solution (16 g PEG 6000+4.2 g $\mathrm{NaCl}$ in $100 \mathrm{ml}$ deionized water), and stirred overnight at $4{ }^{\circ} \mathrm{C}$. The next day, it was centrifuged at $10,000 \mathrm{rpm}$ at 
$4{ }^{\circ} \mathrm{C}$ for $20 \mathrm{~min}$, and the supernatant was discarded. The pellet was finally dissolved in $300 \mu \mathrm{l}$ of distilled deionized water to prepare a single virus concentrate sample from a single clam.

Viral RNA was then extracted from the concentrated sample using QIAamp Viral RNA Mini Kit and RVs were detected by real-time RT-PCR using primers and probes designed to target the non-structural protein region 3 (NSP3) of RVs: forward primer JVKF (5'-CAG TGGTTGATGCTCAAGAT GGA-3'; positions 17-39), reverse primer JVKR (5'-TCATTGTAA TCATATTGA ATACCCA-3'; positions 147-123), and TaqMan probe JVKP (FAM-5'-ACAACTGCAGCTTCAAAAGAAGWG T-3' Black Hole Quencher; positions 96-72), as described elsewhere (Jothikumar et al., 2009). Vaccine-specific realtime RT-PCR was done for all real-time RT-PCR-positive samples to detect NSP2 and VP6 regions of Rotarix and RotaTeq strains, respectively (Gautam et al., 2014). The real-time RT-PCR was performed for 45 cycles, with a $\mathrm{Ct}$ (cycle threshold) value of 40 as the detection limit.
Amplifications were confirmed using positive (oligo DNA containing target sequences) and negative external amplification controls.

G-genotypes were determined by sequence-based analysis of the VP7 region after conventional RT-PCR (Hoque et al., 2020).

Total 47 stool samples from RV-positive children (tested RV-positive by immunochromatographic-based rapid diagnosis kits in the clinic) were collected from 2 sentinel clinics in Kawasaki city from January 2017 to January 2020 for genotyping at Kawasaki City Institute for Public Health by conventional RT-PCR followed by sequenced based analysis as described elsewhere (Hoque et al., 2020).

\section{Results}

As shown in Table 1, twenty-four clams were collected per study month and investigated for wild-type RVs and RVvaccine strains. Among total 96 clams, wild-type RVs were

Table 1 Cycle threshold $(\mathrm{Ct})$ values of real-time PCR for RV detection in river clams

\begin{tabular}{|c|c|c|c|c|c|c|c|c|c|c|c|c|}
\hline \multirow[t]{2}{*}{ Serial } & \multicolumn{3}{|l|}{ July, 2019} & \multicolumn{3}{|c|}{ September, 2019} & \multicolumn{3}{|c|}{ November, 2019} & \multicolumn{3}{|c|}{ January, 2020} \\
\hline & Wild-type & RotaTeq & Rotarix & Wild-type & RotaTeq & Rotarix & Wild-type & RotaTeq & Rotarix & Wild-type & RotaTeq & Rotarix \\
\hline 1 & 33.1 & - & - & 35.4 & - & - & 32 & - & - & 33.5 & - & - \\
\hline 2 & 31.6 & - & - & - & - & - & 34.6 & - & - & 31.9 & - & - \\
\hline 3 & 32.1 & - & - & - & - & - & 32.6 & - & - & - & - & - \\
\hline 4 & 32 & - & - & 35.4 & - & - & 31.8 & - & - & 32.6 & - & - \\
\hline 5 & 33.2 & - & - & 35.4 & - & - & 33.9 & - & - & 32.3 & - & - \\
\hline 6 & - & - & - & - & - & - & 33.1 & 36 & - & - & - & - \\
\hline 7 & 30.8 & - & - & - & - & - & 33.6 & - & - & 34.5 & - & - \\
\hline 8 & 31.4 & - & - & - & - & - & - & - & - & 35.5 & - & - \\
\hline 9 & 32 & - & - & - & - & - & 34.3 & - & - & 36.5 & - & - \\
\hline 10 & 32.6 & - & - & - & - & - & 34.9 & - & - & - & - & - \\
\hline 11 & 31.1 & - & - & - & - & - & - & - & - & 35.7 & - & - \\
\hline 12 & 32.8 & - & - & 35 & - & - & 33.9 & - & - & - & - & - \\
\hline 13 & 31.7 & - & - & 35.3 & - & - & 35.4 & - & - & - & - & - \\
\hline 14 & 30.9 & - & - & 35.1 & - & - & 32.7 & - & - & - & - & - \\
\hline 15 & 32.4 & - & - & - & - & - & 34.1 & - & - & 36 & - & - \\
\hline 16 & 31.8 & - & - & - & - & - & 33.7 & - & - & 35.1 & - & - \\
\hline 17 & 32.3 & - & - & - & - & - & 36 & - & - & - & - & - \\
\hline 18 & 32 & - & - & - & - & - & 35.8 & - & - & - & - & - \\
\hline 19 & 35 & - & - & 35.1 & - & - & 34.8 & - & - & - & - & - \\
\hline 20 & - & - & - & - & - & - & - & - & - & - & - & - \\
\hline 21 & 32.2 & - & - & 34.4 & - & - & 33.9 & - & - & 33.3 & - & - \\
\hline 22 & 32.9 & - & - & 32.9 & - & - & - & - & - & 33.4 & - & - \\
\hline 23 & 32 & - & - & - & - & - & - & - & - & - & - & - \\
\hline 24 & 34 & - & - & - & - & - & - & - & - & 35.2 & - & - \\
\hline $\begin{array}{l}\text { Number of } \\
\text { RV-positives } \\
(\%)\end{array}$ & $22(91.7)$ & 0 & 0 & $9(37.5)$ & 0 & 0 & $18(75.0)$ & $1(4.1)$ & 0 & $13(51.2)$ & 0 & 0 \\
\hline
\end{tabular}


detected in 62 (64.6\%): the maximum $91.7 \%$ (22 out of 24 clams, average $\mathrm{Ct}$ value \pm standard deviation was $32.2 \pm 0.9$ ) clams were detected RV-positive in July 2019 followed by $75 \%$ (18 out of $24, \mathrm{Ct}: 33.95 \pm 1.2$ ) in November 2019 , $51.2 \%$ (13 out of $24, \mathrm{Ct}: 34.2 \pm 1.5$ ) in January 2020, and $37.5 \%$ (9 out of $24, \mathrm{Ct}: 34.8 \pm 0.8$ ) in September 2019. However, the detection of vaccine strain did not correlate with the number of wild-type viruses detected. Single clam positive for RotaTeq vaccine virus was detected in November 2019 (Table 1) with $C$ t value 36 indicating clearly positive. The copy number was separately evaluated in this clam and determined 1-2 copies/clam. No Rotarix vaccine strain was detected in the study.

G-genotyping analysis suggested that RVs detected in clams were of G1, G2, G3, G8, and G10 genotypes (Fig. 1). To find out the relationship between RVs in clams and gastroenteritis cases, we collected the information of the last few years of RV detection in the children of nearby Kawasaki City Institute for Public Health. As shown in Fig. 1, the RV season in clinical samples existed from February to June, while RVs were detected in clams from July to January (Table 1). The most frequent genotype in clams were G2 (6 out of 14, 42.9\%), followed by G8 (4 out of 14, 28.6\%), G3 ( 2 out of 14, 14.3\%), G1 (1 out of 14, 7.1\%) and G10 (1 out of $14,7.1 \%)$. Interestingly, similar genotypic distribution was observed in the clinical samples of nearby clinics when investigated from 2017 to 2020: G2 (16 out of 47, 34.0\%), followed by G8 (14 out of 47, 29.8\%), G3 (10 out of 47, $21.3 \%$ ), G9 (4 out of $47,8.5 \%$ ) and G1 (3 out of $47,6.4 \%$ ). Thus, the predominating genotypes in clams and clinical samples of the last few years seem associated. However, the genotypes predominated in the clinical samples in that particular study year (2019-2020) were G8 (13 out of 22, $59.1 \%$ ) followed by G3 (4 out of 22, 18.2\%), G9 (3 out of 22 , $13.6 \%), \mathrm{G} 1$ ( 1 out of $22,4.5 \%)$ and $\mathrm{G} 2$ ( 1 out of $22,4.5 \%)$, not looked properly associated with the genotypes in clams.

\section{Discussion}

The aim of this study was to detect RVs, both wild-type and vaccine strains, in shellfish and correlate the genotypes of RVs detected in shellfish with that of gastroenteritis cases

A RV genotypes detected in clams

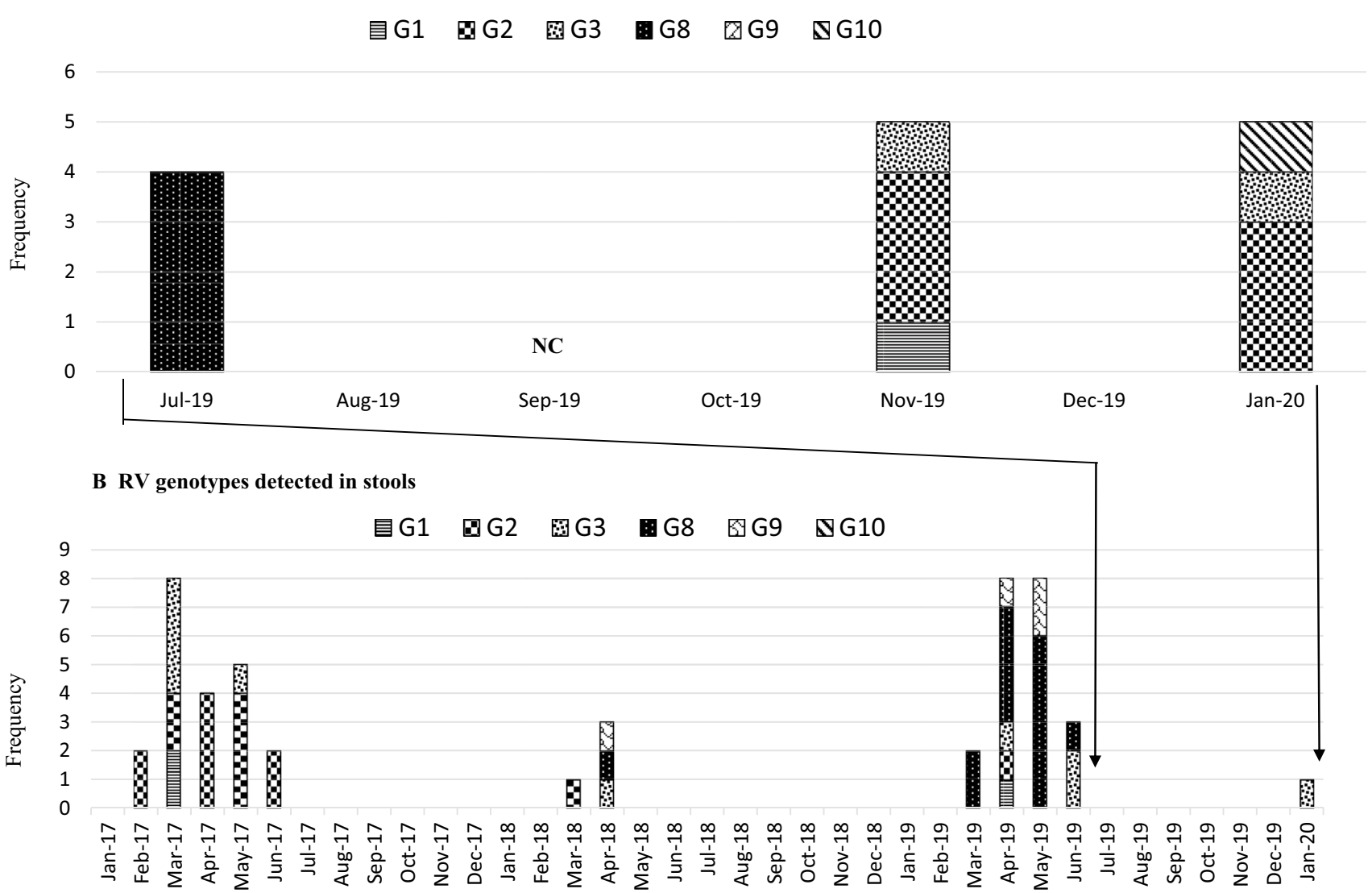

Fig. 1 Distribution of RV genotypes in clams (A) and stools (B). NC not clear for sequenced based genotyping 
of nearby clinics to understand the transmission/circulation of these pathogens in the environment.

Here, we detected RVs from freshwater clams harvested from nature. Herein, clams were collected with 2 months' intervals from July 2019 to January 2020. Although this study was planned to continue at least one year to understand the year-round scenario of RV's accumulation in clams, the COVID-19 pandemic situation halted the study in midway and existing data suggested that wild-type RVs were detected in clams in these months also (Table 1) although RV season in Japan is considered from February to May with a peak in April (Hoque et al., 2020). The detection of RVs in clams in all detection months (July, September, November, and January) suggested that RVs may remain present in shellfish in all seasons including the off-season of RV infection. Recent evidence suggested that the concentration of RVs in oysters was significantly correlated with that in sewage with a lag-time of -6 to 0 weeks which is required for viral transportation from wastewater treatment plants to oysters (Ito et al., 2021).

Detection of RotaTeq vaccine strain in clams remained another major finding of the present study. Very recently, the detection of RV-vaccine strains in oysters has been shown for the first time by Ito et al. (2021). According to their findings, the positive rates of wild-type RVA, RotaTeq, and Rotarix in oysters were 54, 31, and $14 \%$, respectively, which remained comparable with those of wild-type RVA (57\%), RotaTeq (35\%), and Rotarix (0\%) in sewage water (Ito et al., 2021). In the present study, we detected wild-type RV (64.6\%), RotaTeq (1\%) but not Rotarix (0\%) viruses in freshwater clams. The detection rate may have varied due to the differences in the methodology and sensitivity of primers. Here, we did not use the pooling method because we aimed to detect wild-type/vaccine RV strain from individual clams. The primers and probes used here for selective detection of Rotarix and RotaTeq vaccine strains were very specific to Rotarix NSP2 and RotaTeq VP6 genes, respectively, that were developed and validated by Gautam et al. (2014) exhibiting 100\% sensitivity, 98.6-99\% specificity, 92.5-96\% PPV, 99-100\% NPV with 91-94\% efficiency and 1-2 copies limit of detection toward selectively detecting target vaccine strains. Here, the $C \mathrm{t}$ value of the single RotaTeq-positive clam was 36 indicating clearly positive and the copy number was evaluated 1-2 copies in the clam. RV-vaccines are used in all months. So, vaccine strain could be found at any month. Nevertheless, vaccine strains were detected fewer than wild-type strains, probably because disposable baby diapers are widely used in developed countries for infants younger than 6 months when vaccine doses are administered.

Herein, RVs were detected in clams by real-time RTPCR as well as by conventional RT-PCR during genotypic analysis. However, conventional RT-PCR remained less sensitive than real-time RT-PCR, and all RV-positive samples did not yield clear amplicons of the VP7 region for sequence-based genotyping. Genotypes detected in clams remained comparable with those detected in AGE cases of nearby clinics of the last few years but not with that particular year only. However, the G10 genotype detected in clams was not found in clinical samples. Thus, the data of genotypic analysis of clams and clinical samples raised several possibilities: 1 . RV strains of previous years may persist in clams for a long time, 2 . strains from asymptomatic infected individuals can be accumulated, 3. RVs, not only from nearby areas but also from a long distance across, may be carried away by river water and accumulate in the clams in bay areas.

Bivalve mollusks like clams, oysters, mussels, and scallops are popularly eaten in different types of foods. Similar to clams, other bivalve mollusks also may remain contaminated similarly in all seasons and may cause the spread of $\mathrm{RV}$ infection from food handlers, or asymptomatic adult carriers to vulnerable children aged $<5$ years.

Bivalve mollusks may contain PCR-inhibitors, therefore, lack of internal process control in the detection technique remained a limitation of this study. Nevertheless, frequent detection of RV-positive clams indicated that the method was sensitive enough to detect RV after minimizing the effect of inhibitors. Lack of quantitative analysis and viability testing remained some other limitations of this study.

In conclusion, this short communication remains very important to understand the spread of RVs. Our data suggest that RVs from infected patients, either symptomatic or asymptomatic, or from long distances can be accumulated in bivalve mollusks and can persist for a long time. The shellfish of off-season also may remain contaminated with RVs and may remain responsible for the next seasonal RV outbreak. Furthermore, we provided evidence of the accumulation of vaccine strains in bivalve mollusks that pose a threat of unexpected infections and virus genomic reassortments. Although the detection rate of vaccine strains in clams remained low in Japan, it may vary in other countries. Further studies are required to understand the viability and whether this bioaccumulation and bioconcentration of bivalve mollusks have any stimulating role in genetic reassortment.

Acknowledgements This work was supported by Grant-in-Aid for Public Foundation of the Vaccination Research Center (2018-38, 2019-41, 2020-39) and Japan Agency for Medical Research and Development (AMED: JP20fk0108122 and 20fk0108122s0202).

Data Availability Not applicable.

\section{Declarations}

Conflict of interest We do not have any association either directly or indirectly that might pose a conflict of interest. 


\section{References}

Anderson, E. J. (2008). Rotavirus vaccines: Viral shedding and risk of transmission. The Lancet Infectious Diseases, 8(10), 642-649. https://doi.org/10.1016/S1473-3099(08)70231-7

Anses. (2012, April). Rotavirus. Retrieved June 6, 2021, from https:// www.anses.fr/en/system/files/MIC2012sa0031FiEN.pdf

Araki, K., Hara, M., Sakanishi, Y., Shimanoe, C., Nishida, Y., Matsuo, M., \& Tanaka, K. (2016). Estimating rotavirus vaccine effectiveness in Japan using a screening method. Human Vaccines \& amp; Immunotherapeutics, 12(5), 1244-1249. https://doi.org/10.1080/ 21645515.2015.1121337

Bucardo, F., Rippinger, C. M., Svensson, L., \& Patton, J. T. (2012). Vaccine-derived NSP2 segment in rotaviruses from vaccinated children with gastroenteritis in Nicaragua. Infection, Genetics and Evolution, 12(6), 1282-1294. https://doi.org/10.1016/j.meegid. 2012.03.007

Burnett, E., Jonesteller, C. L., Tate, J. E., Yen, C., \& Parashar, U. D. (2017). Global impact of rotavirus vaccination on childhood hospitalizations and mortality from diarrhea. Journal of Infectious Diseases, 215(11), 1666-1672. https://doi.org/10.1093/infdis/ jix186

Gautam, R., Esona, M. D., Mijatovic-Rustempasic, S., Ian Tam, K., Gentsch, J. R., \& Bowen, M. D. (2014). Real-time RT-PCR assays to differentiate wild-type group A rotavirus strains from $\operatorname{Rotarix}((\mathrm{R}))$ and $\operatorname{RotaTeq}(\mathrm{R}))$ vaccine strains in stool samples. Human Vaccines \&amp; Immunotherapeutics, 10(3), 767-777. https://doi.org/10.4161/hv.27388

GBD 2016 Diarrhoeal Disease Collaborators. (2018). Estimates of the global, regional, and national morbidity, mortality, and aetiologies of diarrhoea in 195 countries: A systematic analysis for the Global Burden of Disease Study 2016. The Lancet Infectious Diseases, 18(11), 1211-1228. https://doi.org/10.1016/S1473-3099(18) 30362-1

Hoque, S. A., Islam, M. T., Kobayashi, M., Takanashi, S., Anwar, K. S., Watanabe, T., Khamrin, P., Okitsu, S., Hayakawa, S., \& Ushijima, H. (2018a). Our response to the letter to the editor. Vaccine, 36(34), 5110-5111. https://doi.org/10.1016/j.vaccine.2018.05.004

Hoque, S. A., Kobayashi, M., Takanashi, S., Anwar, K. S., Watanabe, T., Khamrin, P., Okitsu, S., Hayakawa, S., \& Ushijima, H. (2018b). Role of rotavirus vaccination on an emerging G8P[8] rotavirus strain causing an outbreak in central Japan. Vaccine, 36(1), 43-49. https://doi.org/10.1016/j.vaccine.2017.11.056

Hoque, S. A., Iizuka, I., Kobayashi, M., Takanashi, S., Anwar, K. S., Islam, M. T., Khamrin, P., Okitsu, S., Hayakawa, S., \& Ushijima, H. (2019a). Determining effectiveness of rotavirus vaccine by immunochromatography and reverse transcriptase polymerase chain reaction: A comparison. Vaccine, 37(39), 5886-5890. https://doi.org/10.1016/j.vaccine.2019.07.091

Hoque, S. A., Thongprachum, A., Takanashi, S., Mostafa, S. M., Saito, H., Anwar, K. S., Nomura, A., Begum, R., Sultana, U. N., Hossain, T., Khamrin, P., Okitsu, S., Hayakawa, S., \& Ushijima, H. (2019b). Alarming situation of spreading enteric viruses through sewage water in Dhaka city: Molecular epidemiological evidences. Food and Environmental Virology, 11(1), 65-75. https:// doi.org/10.1007/s12560-018-09363-Z

Hoque, S. A., Khandoker, N., Thongprachum, A., Khamrin, P., Takanashi, S., Okitsu, S., Nishimura, S., Kikuta, H., Yamamoto, A., Sugita, K., Baba, T., Kobayashi, M., Hayakawa, S., Mizuguchi, M., Maneekarn, N., \& Ushijima, H. (2020). Distribution of rotavirus genotypes in Japan from 2015 to 2018: Diversity in genotypes before and after introduction of rotavirus vaccines. Vaccine, 38(23), 3980-3986. https://doi.org/10.1016/j.vaccine.2020.03.061

Ito, E., Pu, J., Miura, T., Kazama, S., Nishiyama, M., Ito, H., Konta, Y., Omura, T., \& Watanabe, T. (2021). Detection of rotavirus vaccine strains in oysters and sewage and their relationship with the gastroenteritis epidemic. Applied and Environmental Microbiology, 87(10), 55. https://doi.org/10.1128/AEM.02547-20

JFHA. (2015). Food hygiene inspection guidelines $=$ Standard methods of analysis in food safety regulation. Japan Food Hygiene Association.

JHI. (2021). Vaccination. Retrieved September 28, 2021, from https:// japanhealthinfo.com/child-health-and-childcare/vaccination/

Jothikumar, N., Kang, G., \& Hill, V. R. (2009). Broadly reactive TaqMan assay for real-time RT-PCR detection of rotavirus in clinical and environmental samples. Journal of Virological Methods, 155(2), 126-131. https://doi.org/10.1016/j.jviromet.2008.09.025

Kawata, K., Hoque, S. A., Nishimura, S., Yagyu, F., Islam, M. T., Sharmin, L. S., Pham, N. T. K., Onda-Shimizu, Y., Quang, T. D., Takanashi, S., Okitsu, S., Khamrin, P., Maneekarn, N., Hayakawa, S., \& Ushijima, H. (2021). Role of rotavirus vaccination on G9P [8] rotavirus strain during a seasonal outbreak in Japan. Human Vaccines \&amp; Immunotherapeutics. https://doi.org/10. 1080/21645515.2021.1925060

Keller, R., Pratte-Santos, R., Scarpati, K., Martins, S. A., Loss, S. M., Fumian, T. M., Miagostovich, M. P., \& Cassini, S. T. (2019). Surveillance of enteric viruses and thermotolerant coliforms in surface water and bivalves from a Mangrove Estuary in Southeastern Brazil. Food and Environmental Virology, 11(3), 288-296. https://doi.org/10.1007/s12560-019-09391-3

Kittigul, L., Panjangampatthana, A., Rupprom, K., \& Pombubpa, K. (2014). Genetic diversity of rotavirus strains circulating in environmental water and bivalve shellfish in Thailand. International Journal of Environmental Research and Public Health, 11(2), 1299-1311. https://doi.org/10.3390/ijerph110201299

Meghnath, K., Hasselback, P., McCormick, R., Prystajecky, N., Taylor, M., McIntyre, L., Man, S., Whitfield, Y., Warshawsky, B., McKinley, M., Bitzikos, O., Hexemer, A., Galanis, E., \& Outbreak, T. (2019). Outbreaks of norovirus and acute gastroenteritis associated with British Columbia Oysters, 2016-2017. Food and Environmental Virology, 11(2), 138-148. https://doi.org/10.1007/ s12560-019-09374-4

Patel, N. C., Hertel, P. M., Estes, M. K., de la Morena, M., Petru, A. M., Noroski, L. M., Revell, P. A., Hanson, I. C., Paul, M. E., Rosenblatt, H. M., \& Abramson, S. L. (2010). Vaccine-acquired rotavirus in infants with severe combined immunodeficiency. New England Journal of Medicine, 362(4), 314-319. https://doi.org/ 10.1056/NEJMoa0904485

Quiroz-Santiago, C., Vazquez-Salinas, C., Natividad-Bonifacio, I., Barron-Romero, B. L., \& Quinones-Ramirez, E. I. (2014). Rotavirus G2P[4] detection in fresh vegetables and oysters in Mexico City. Journal of Food Protection, 77(11), 1953-1959. https://doi. org/10.4315/0362-028X.JFP-13-426

Richards, G. P. (2016). Shellfish-associated enteric virus illness: Virus localization, disease outbreaks and prevention. Viruses in Foods. https://doi.org/10.1007/978-3-319-30723-7_7

Snelling, T. L., Andrews, R. M., Kirkwood, C. D., Culvenor, S., \& Carapetis, J. R. (2011). Case-control evaluation of the effectiveness of the G1P[8] human rotavirus vaccine during an outbreak of rotavirus G2P[4] infection in central Australia. Clinical Infectious Diseases, 52(2), 191-199. https://doi.org/10.1093/cid/ciq101

Stanford Children's Health. (2021). What Is Rotavirus?, Retrieved June 6, 2021, from https://www.stanfordchildrens.org/en/topic/default? id=what-is-rotavirus-1-2802

Tate, J. E., Burton, A. H., Boschi-Pinto, C., Steele, A. D., Duque, J., \& Parashar, U. D. (2012). 2008 estimate of worldwide rotavirusassociated mortality in children younger than 5 years before the introduction of universal rotavirus vaccination programmes: A systematic review and meta-analysis. The Lancet Infectious Diseases, 12(2), 136-141. https://doi.org/10.1016/S1473-3099(11) 70253-5 
Thongprachum, A., Fujimoto, T., Takanashi, S., Saito, H., Okitsu, S., Shimizu, H., Khamrin, P., Maneekarn, N., Hayakawa, S., \& Ushijima, H. (2018). Detection of nineteen enteric viruses in raw sewage in Japan. Infection, Genetics and Evolution. https://doi. org/10.1016/j.meegid.2018.05.006

Upfold, N. S., Luke, G. A., \& Knox, C. (2021). Occurrence of human enteric viruses in water sources and shellfish: A focus on Africa. Food and Environmental Virology, 13(1), 1-31. https://doi.org/ 10.1007/s12560-020-09456-8

Victoria, M., Tort, L. F., Garcia, M., Lizasoain, A., Maya, L., Leite, J. P., Miagostovich, M. P., Cristina, J., \& Colina, R. (2014). Assessment of gastroenteric viruses from wastewater directly discharged into Uruguay River, Uruguay. Food and Environmental Virology, 6(2), 116-124. https://doi.org/10.1007/s12560-014-9143-7

Werther, R. L., Crawford, N. W., Boniface, K., Kirkwood, C. D., \& Smart, J. M. (2009). Rotavirus vaccine induced diarrhea in a child with severe combined immune deficiency. The Journal of Allergy and Clinical Immunology, 124(3), 600. https://doi.org/10.1016/j. jaci.2009.07.005

WHO. (2019). Immunization, Vaccines and Biologicals. Retrieved November 4, 2019, from https://www.who.int/immunization/ diseases/rotavirus/en/

Yen, C., Jakob, K., Esona, M. D., Peckham, X., Rausch, J., Hull, J. J., Whittier, S., Gentsch, J. R., \& LaRussa, P. (2011). Detection of fecal shedding of rotavirus vaccine in infants following their first dose of pentavalent rotavirus vaccine. Vaccine, 29(24), 4151-4155.

Publisher's Note Springer Nature remains neutral with regard to jurisdictional claims in published maps and institutional affiliations. 